\title{
ERKLÄRUNG DER ABKÜRZUNGEN
}

Abh $=$ Abhandlungen

AbhSGdWphhkl = Abhandlungen der Sächsischen Gesellschaft der Wissenschaften, philologisch-historische Klasse.

$\mathrm{AdB}=$ Allgemeine deutsche Biographie, hrsg. durch die historische Kommission der Münchener Akademie der Wissenschaften.

AfdA = Anzeiger für deutsches Altertum u. deutsche Literaturgeschichte, hrsg. v. Steinmeyer u. a.

AfKdM = Anzeiger für Kunde des deutschen Mittelalters, hrsg.v. Aufsess u. Mone.

AfKdV = Anzeiger für Kunde der deutschen Vorzeit, hrsg. v. Mone.

Archiv $=$ Archiv für das Studium der neueren Sprachen, hrsg. v. Herrig u. a.

$\mathrm{BdN}=$ Bibliothek der gesamten deutschen Nationalliteratur (Quedlinburg $\mathrm{u}$. Leipzig, Basse).

BerlSB $=$ Sitzungsberichte der $\mathrm{K}$. Preußischen Akademie der Wissenschaften . Berlin.

DGed $=$ Deutsche Gedichte.

Diemer, D.Ged (oder Diemer) = Deutsche Gedichte des 1r. und 12. Jahrh., hrsg. v. Jos. Diemer. Wien 1849 .

$\mathrm{DHB}=$ Deutsches Heldenbuch. 5 Bde. Berlin $\mathrm{t} 866-70$.

$\mathrm{DLZ}=$ Deutsche Literaturzeitung.

DT $=$ Deutsche Texte des Mittelalters, herausg. v. d. Kgl. Preußischen Akademie der Wissenschaften.

DWB $=$ Deutsches Wörterbuch von J. u. W. Grimm.

Fdgr., Fundgr $=$ Fundgruben zur Geschichte deutscher Sprache und Literatur, hrsg. v. H. Hoffmann.

Germ $=$ Germania, Vierteljahrsschrift f. deutsche Altertumskunde, hrsg: v. Pfeiffer, Bartsch.

Germ.Abh. $=$ Germanistische Abhandlungen, hrsg. v. Weinhold, Vogt.

GGA $=$ Göttinger gelehrte Anzeigen.

GGN $=$ Nachrichten von der k. Gesellschaft der Wissenschaften zu Göttingen.

Goedeke $=$ K. Goedeke, Grundriß zur Geschichte der deutschen Dichtung aus den Quellen. 2. Aufl.

GRM = Germanisch-Romanische Monatsschrift, hrsg. v. H. Schröder.

Grundr. $=$ Grundriß der germanischen Philologie, hrsg. v. H. Paul. 2. Aufl.

Hagen, D.Ged. $=$ Deutsche Gedichte des Mittelalters, hrsg. v. v. d. Hagen u. Büsching.

Kelle oder Kelle, $\mathrm{LG}=$ Geschichte der deutschen Literatur von der ältesten Zeit bis z. 13. Jhrh. v. Joh. Kelle.

Kürschner $=$ Deutsche Nationalliteratur, historisch-kritische Ausgabe, hrsg. v. Kürschner.

$\mathrm{LCbl}=$ Literarisches Centralblatt.

Lbl., Literaturbl. $=$ Literaturblatt für germanische $u$. romanische Philologie, hrsg. v. Behaghel u. Neumann.

LG $=$ Literaturgeschichte.

Lit. Ver. $=$ Bibliothek des Literarischen Vereins i. Stuttgart.

$\mathrm{MA}=$ Mittelalter.

Maßmann (D. Ged.) = Deutsche Gedichte des 12. Jahrhunderts, hrsg. v. H. F. Massmann. 
$M F=$ Des Minnesangs Frühling, hrsg. v. Lachmann u. Haupt.

MFV = Des Minnesangs Frühling, neu bearbeitet von F. Vogt. 3. Aufl. 1920.

$M G=$ Monumenta Germaniae Historica; SS = Scriptores.

MSD = Denkmäler deutscher Poesie u. Prosa aus dem 8. bis 12. Jahrhundert, hrsg. v. Müllenhoff u. Scherer. 3. Ausg. v. Steinmeyer 1892.

$\mathrm{MSH}=$ Minnesinger. Deutsche Liederdichter des 12., I3. u. 14. Jahrhunderts, gesammelt von F. H. v. d. Hagen.

Münchener $\mathrm{SB}=$ Sitzungsberichte der philosophisch-philologischen u. der historischen Klasse der K. B. Akademie der Wissenschaften zu München.

Ndr. = Neudrucke deutscher Literaturwerke des 16. u. 17. Jahrhunderts, hrsg. v. W. Braune.

Neue Jahrb. (N. Jahrb.) = Neue Jahrbücher für das klassische Altertum, Geschichte und deutsche Literatur und für Pädagogik.

$\mathrm{Pal} .=\mathrm{Palästra}$. Untersuchungen und Texte aus der deutschen $\mathrm{u}$. englischen Philologie, hrsg. v. Brandl u. Schmidt.

$\mathrm{PBB}=$ Beiträge zur Geschichte der deutschen Sprache u. Literatur, hrsg. v. H. Paul u. W. Braune.

Petzet-Glauning $=$ Deutsche Schrifttafeln des 9. bis 16. Jahrhunderts von E. Petzet u. O. Glauning.

Prager DSt. $=$ Prager Deutsche Studien, hrsg. v. Kraus u. Sauer.

$\mathrm{QF}=$ Quellen $u$. Forschungen zur Sprache u. Kulturgeschichte der german. Völker, hrsg. v. Ten Brink, Scherer u. a.

Rhein. Beitr. = Rheinische Beiträge u. Hilfsbücher zur germanischen Philclogie u. Volkskunde, hrsg. v. Frings, Meißner, Müller.

Romania = Romania. Recueil trimestrel consacré à l'étude des langues et des littératures Romanes.

Rom. Forsch. = Romanische Forschungen, hrsg. v. Vollmöller.

Rom. Jahresber. $=$ Kritischer Jahresbericht über die Fortschritte der romanischen Philologie, hrsg. v. Vollmöller.

$\mathrm{SB}=$ Sitzungsberichte.

Unwerth-Siebs = Geschichte der deutschen Literatur bis zur Mitte des I1. Jahrhunderts von W. v. Unwerth $u$. Th. Siebs (Grundriß der deutschen Literaturgeschichte $\mathrm{I}$ ).

Waag $=$ Kleinere deutsche Gedichte des II. u. I2. Jahrhunderts, hrsg. v. A. Waag. 2. Auf.. 1916.

Westd. Zs. = Westdeutsche Zeitschrift für Geschichte u. Kunst.

WSB oder Wiener SB = Sitzungsberichte der kais. Akademie der Wissenschaften in Wien, philos.-histor. Klasse.

$\mathrm{Z}$ oder $\mathrm{Zs}=$ Zeitschrift.

ZfdA = Zeitschrift für deutsches Altertum, hrsg. v. Haupt u. a.

$\mathrm{ZfdPh}=$ Zeitschrift für deutsche Philologie, hrsg. v. Höpfner, Zacher u. a.

Zfd Mundarten $=$ Zeitschrift für deutsche Mundarten.

ZfdU $=$ Zeitschrift für den deutschen Unterricht, hrsg. v. Hildebrand, Lyon u. a.

Zfd Wortf. = Zeitschrift für deutsche Wortforschung.

$\mathrm{ZfrPh}=$ Zeitschrift für romanische Philologie.

ZffrzSpr = Zeitschrift für französische Sprache u. Literatur. 